\title{
Structural Properties of Twin-Free Graphs
}

\author{
Irène Charon \\ GET - Télécom Paris \& CNRS - LTCI UMR 5141 \\ 46, rue Barrault, 75634 Paris Cedex 13 - France \\ charon@infres.enst.fr \\ Iiro Honkala \\ Department of Mathematics - University of Turku \\ FIN-20014 Turku - Finland \\ honkala@utu.fi \\ Olivier Hudry \\ GET - Télécom Paris \& CNRS - LTCI UMR 5141 \\ 46, rue Barrault, 75634 Paris Cedex 13 - France \\ hudry@infres.enst.fr \\ Antoine Lobstein \\ CNRS - LTCI UMR 5141 \& GET - Télécom Paris \\ 46, rue Barrault, 75634 Paris Cedex 13 - France \\ lobstein@infres.enst.fr
}

Submitted: Jan 31, 2006; Accepted: Dec 21, 2006; Published: Jan 29, 2007

Mathematics Subject Classification: 05C75, 05C35

Key Words: Graph Theory, Identifying Codes, Trees, Paths

\begin{abstract}
Consider a connected undirected graph $G=(V, E)$, a subset of vertices $C \subseteq V$, and an integer $r \geq 1$; for any vertex $v \in V$, let $B_{r}(v)$ denote the ball of radius $r$ centered at $v$, i.e., the set of all vertices linked to $v$ by a path of at most $r$ edges. If for all vertices $v \in V$, the sets $B_{r}(v) \cap C$ are all nonempty and different, then we call $C$ an $r$-identifying code. A graph admits at least one $r$-identifying code if and only if it is $r$-twin-free, that is, the sets $B_{r}(v), v \in V$, are all different.

We study some structural problems in $r$-twin-free graphs, such as the existence of the path with $2 r+1$ vertices as a subgraph, or the consequences of deleting one vertex.
\end{abstract}




\section{Introduction}

Given a connected, undirected, finite graph $G=(V, E)$ and an integer $r \geq 1$, we define $B_{r}(v)$, the ball of radius $r$ centered at $v \in V$, by

$$
B_{r}(v)=\{x \in V: d(x, v) \leq r\}
$$

where $d(x, v)$ denotes the number of edges in any shortest path between $v$ and $x$.

Whenever $d(x, v) \leq r$, we say that $x$ and $v r$-cover each other (or simply cover if there is no ambiguity). A set $X \subseteq V$ covers a set $Y \subseteq V$ if every vertex in $Y$ is covered by at least one vertex in $X$.

Two vertices $v_{1}, v_{2} \in V$ such that $B_{r}\left(v_{1}\right)=B_{r}\left(v_{2}\right)$ are called $r$-twins or twins. If $G$ has no $r$-twins, that is, if

$$
\forall v_{1}, v_{2} \in V\left(v_{1} \neq v_{2}\right), B_{r}\left(v_{1}\right) \neq B_{r}\left(v_{2}\right),
$$

then we say that $G$ is $r$-twin-free or twin-free.

A graph with one vertex is trivially twin-free, and generally we consider graphs with at least two vertices.

Twin-free graphs are of interest because they are strongly connected with identifying codes, which we now define.

A code $C$ is a nonempty set of vertices, and its elements are called codewords. For each vertex $v \in V$, we denote by

$$
K_{C, r}(v)=C \cap B_{r}(v)
$$

the set of codewords which $r$-cover $v$. Two vertices $v_{1}$ and $v_{2}$ with $K_{C, r}\left(v_{1}\right) \neq K_{C, r}\left(v_{2}\right)$ are said to be $r$-separated, or separated, by code $C$.

A code $C$ is called $r$-identifying, or identifying, if the sets $K_{C, r}(v), v \in V$, are all nonempty and distinct [11]. In other words, all vertices must be covered and pairwise separated by $C$.

Remark 1. For given $G=(V, E)$ and integer $r$, the graph $G$ admits at least one $r$ identifying code if and only if it is $r$-twin-free. Indeed, if for all $v_{1}, v_{2} \in V, B_{r}\left(v_{1}\right)$ and $B_{r}\left(v_{2}\right)$ are different, then $C=V$ is $r$-identifying. Conversely, if for some $v_{1}, v_{2} \in V$, $B_{r}\left(v_{1}\right)=B_{r}\left(v_{2}\right)$, then for any code $C \subseteq V$, we have $K_{C, r}\left(v_{1}\right)=K_{C, r}\left(v_{2}\right)$. This is why $r$-twin-free graphs are also called $r$-identifiable. For instance, there is no $r$-identifying code in a complete graph (or clique) with at least two vertices.

Remark 2. If $G$ is not connected, we simply consider each of its connected components, and apply the above definitions.

We recall that an induced subgraph of $G=(V, E)$ is a graph $G_{1}=\left(V_{1}, E_{1}\right)$ where $V_{1} \subseteq V$ and $E_{1}$ is the set of edges in $E$ which have both ends in $V_{1}$, whereas a subgraph is a graph $G_{2}=\left(V_{2}, E_{2}\right)$ where $V_{2} \subseteq V$ and $E_{2}$ is included in the set of edges in $E$ which have both ends in $V_{2}$. 
For $X \subseteq V$, we denote by $G_{X}$ the induced subgraph with vertex set $V \backslash X$, and for $x \in V$, we set $G_{x}=G_{\{x\}}$.

In the following, $n$ will denote the number of vertices of $G$. For any integer $q>0, P_{q}$ will denote the path on $q$ vertices, and the length of $P_{q}$ will be equal to $q-1$, its number of edges. Moreover, if $v_{1}, v_{2}, \ldots, v_{q}$ denote the vertices in $P_{q}$, we shall assume that these vertices are numbered in such a way that the edges in $P_{q}$ are $\left\{v_{i}, v_{i+1}\right\}$ for $1 \leq i<q$. The cycle of length $q, \mathcal{C}_{q}$, with $q$ vertices and $q$ edges, consists of $P_{q}$ to which we add the edge $\left\{v_{q}, v_{1}\right\}$.

The motivations for identifying codes come, for instance, from fault diagnosis in multiprocessor systems. Such a system can be modeled as a graph where vertices are processors and edges are links between processors. Assume that at most one of the processors is malfunctioning and we wish to test the system and locate the faulty processor. For this purpose, some processors (constituting the code) will be selected and assigned the task of testing their neighbourhoods (i.e., the vertices at distance at most $r$ ). Whenever a selected processor (i.e., a codeword) detects a fault, it sends an alarm signal, saying that one element in its neighbourhood is malfunctioning, and we require that we can uniquely tell the location of the malfunctioning processor based only on the information which ones of the codewords gave the alarm.

Identifying codes were introduced in [11], and they constitute now a topic of their own, studied in a large number of various papers, investigating particular graphs or families of graphs (such as certain infinite regular grids, trees, chains, cycles, or the $k$-cube), dealing with complexity issues, or using heuristics such as the noising methods for the construction of small codes. See, e.g., [2], [3], [4], [5], [6], [10], [13], and references therein, or [14].

In Section 2, we show that any connected $r$-twin-free graph contains the path $P_{2 r+1}$ as a subgraph; we conjecture that any connected $r$-twin-free graph contains the path $P_{2 r+1}$ as an induced subgraph (and we prove this for the path $P_{r+2}$ ).

In Section 3, we study the consequences of the deletion of a vertex in a connected $r$-twin-free graph; the results differ according to the values of $r$. In particular, we prove that all connected $r$-twin-free graphs remain $r$-twin-free after deleting one appropriate vertex when $r=1$, and that the same is true for all trees, except $P_{2 r+1}$.

Some of these results were already stated without proofs in [7].

\section{The existence of $P_{2 r+1}$ in $r$-twin free graphs}

In this section, we prove that any connected $r$-twin-free graph $G$ contains $P_{2 r+1}$ as a subgraph, for all $r \geq 1$. We conjecture that $G$ even contains $P_{2 r+1}$ as an induced subgraph, and prove it for $P_{r+2}$.

Theorem 1 Let $r \geq 1$ and $G$ be any connected $r$-twin-free graph with at least two vertices. Then $P_{2 r+1}$ is a subgraph of $G$. 

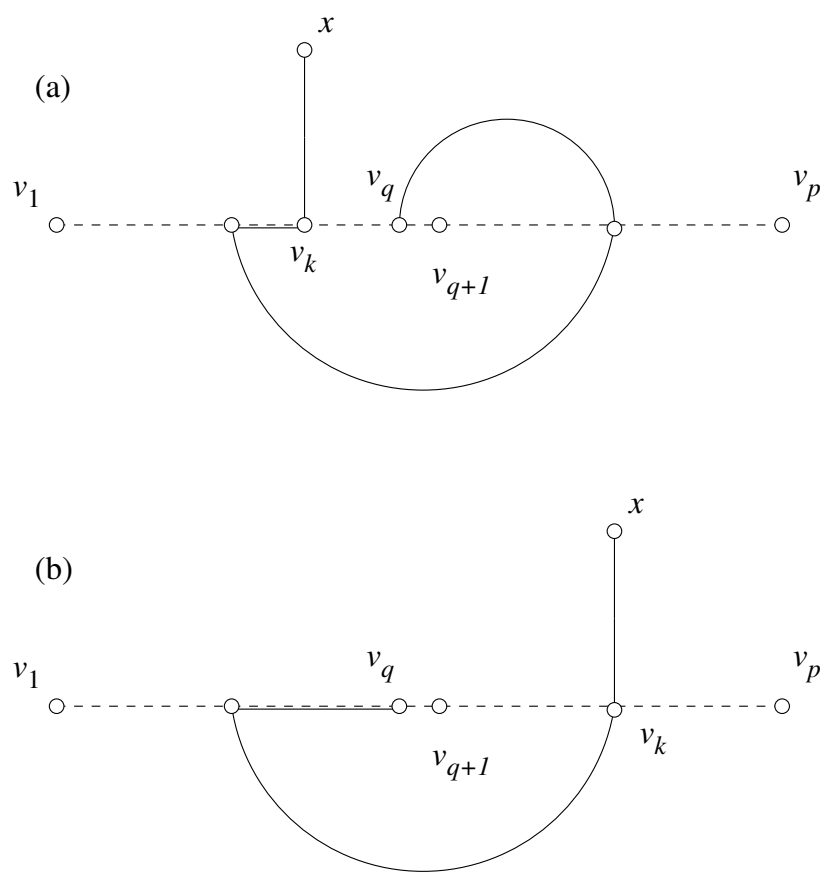

Figure 1: The path $P^{*}$ is in dashed line, the path $P_{r+1}$ is in plain line.

Proof. Let $G$ fulfill the conditions of Theorem 1. Consider a longest path $P^{*}$ of $G$, with $p$ vertices, $v_{1}, v_{2}, \ldots, v_{p}$, and assume that $p \leq 2 r$. We set $q=\lfloor p / 2\rfloor$.

Since $G$ is $r$-twin-free, for any two vertices $w$ and $z$, there is at least one vertex which $r$-covers exactly one of them; we shall say that such a vertex separates $w$ and $z$.

It is easy to check that the vertices of $P^{*}$ cannot separate $v_{q}$ and $v_{q+1}$, because the length of $P^{*}$ is too small. Therefore, there is a vertex $x \notin P^{*}$ which separates $v_{q}$ and $v_{q+1}$. Then two cases occur.

Case (1): $x$ covers $v_{q}$, not $v_{q+1}$.

Thus we have $d\left(x, v_{q}\right) \leq r$ and $d\left(x, v_{q+1}\right) \geq r+1$. Since $v_{q}$ and $v_{q+1}$ are adjacent, we have $d\left(x, v_{q}\right)=r$ and $d\left(x, v_{q+1}\right)=r+1$. Let $P_{r+1}$ be a path of length $r$ between $x$ and $v_{q}$. The paths $P^{*}$ and $P_{r+1}$ have at least $v_{q}$ in common. Let $v_{k}$ be the vertex belonging simultaneously to $P^{*}$ and $P_{r+1}$, and which is the closest to $x$ (see Figure 1 ): the vertices of $P_{r+1}$ between $x$ (included) and $v_{k}$ (excluded) do not belong to $P^{*}$. We have now two subcases, according to the value of $k$ with respect to $q$.

Subcase (1.a): $1 \leq k \leq q$ :

$v_{k}$ is on $P^{*}$ between $v_{1}$ and $v_{q}$ (see Figure 1.a). Consider the path $P$ obtained by the concatenation of the part of $P_{r+1}$ between $x$ and $v_{k}$ and the part of $P^{*}$ between $v_{k}$ and $v_{p}$ (note that, thanks to the definition of $v_{k}$, there is no cycle and $P$ is indeed a path; this will also hold in all other cases). The length of $P$ is at least $r+p-q=r+\lceil p / 2\rceil$ (there are $r$ edges from $x$ to $v_{q}$ and $p-q$ edges from $v_{q}$ to $v_{p}$ ); hence a contradiction: $P$ would be longer than $P^{*}$ (the length of $P^{*}$ is equal to $p-1$ and we assumed that $p$ is less than 

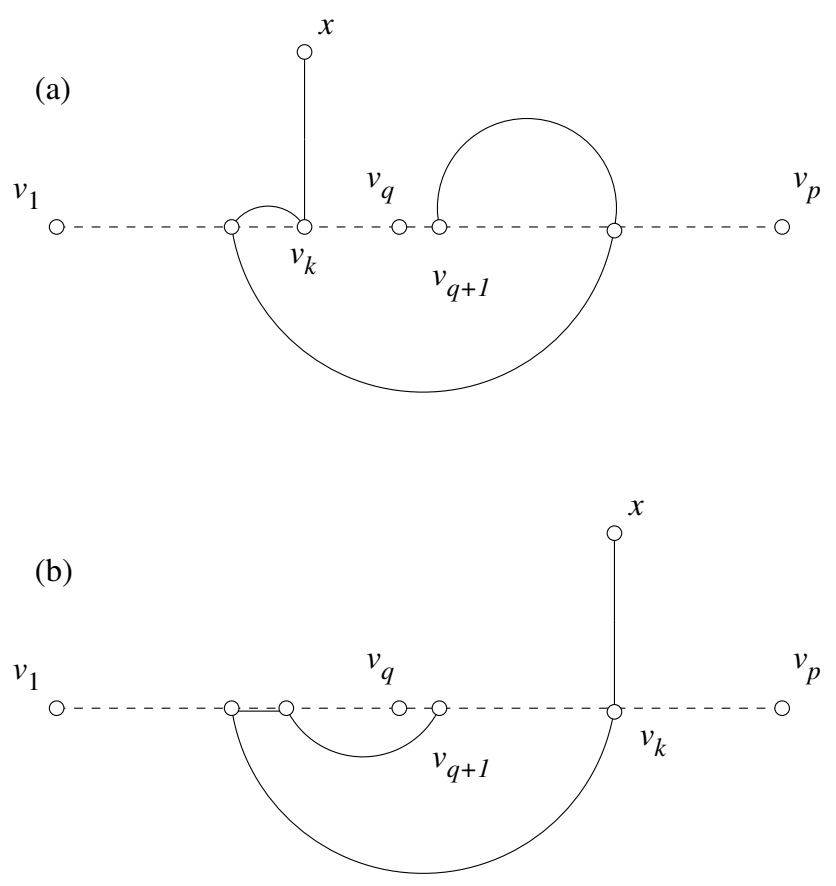

Figure 2: The path $P^{*}$ is in dashed line, the path $P_{r+1}$ is in plain line.

$2 r+1)$.

Subcase (1.b): $q+1 \leq k \leq p$ :

Now $v_{k}$ is on $P^{*}$ between $v_{q+1}$ and $v_{p}$ (see Figure 1.b; it is obvious that $k$ cannot be equal to $q+1$, but this does not matter). Consider the path $P$ obtained by the concatenation of the part of $P^{*}$ going from $v_{1}$ to $v_{k}$ and the part of $P_{r+1}$ going from $v_{k}$ to $x$. The length of $P$ is greater than or equal to $q+r+1$ : there are $q$ edges from $v_{1}$ to $v_{q+1}$ and at least $r+1$ edges from $v_{q+1}$ to $x$, since $d\left(x, v_{q+1}\right)=r+1$. So $P$ is longer than $P^{*}$, a contradiction with the definition of $P^{*}$.

Case (2): $x$ covers $v_{q+1}$, not $v_{q}$.

As $v_{q}$ and $v_{q+1}$ play similar roles if $p$ is even, we may assume that $p$ is odd; otherwise, see Case (1). Hence, $p=2 q+1$. Similarly to Case (1), we have $d\left(x, v_{q}\right)=r+1$ and $d\left(x, v_{q+1}\right)=r$. As above, let $P_{r+1}$ be a path of length $r$ between $x$ and $v_{q+1}$ and let $v_{k}$ be the vertex belonging simultaneously to $P^{*}$ and $P_{r+1}$, and which is the closest to $x$ (see Figure 2). Again, we have two subcases, according to the value of $k$ with respect to $q$.

Subcase $(2 . \mathrm{a}): 1 \leq k \leq q$ :

$v_{k}$ is on $P^{*}$ between $v_{1}$ and $v_{q}$ (see Figure 2.a; similarly to Subcase (1.b), $k$ cannot be equal to $q$, but this still does not matter). Consider the path $P$ obtained by the concatenation of the part of $P_{r+1}$ between $x$ and $v_{k}$ and the part of $P^{*}$ between $v_{k}$ and $v_{p}$. Because $d\left(x, v_{q}\right)=r+1$, the length of $P$ is greater than or equal to $r+1+p-q$, which is greater than the length of $P^{*}$, hence a contradiction.

Subcase (2.b): $q+1 \leq k \leq p$ : 
Now $v_{k}$ is on $P^{*}$ between $v_{q+1}$ and $v_{p}$ (see Figure 2.b). Consider the path $P$ obtained by the concatenation of the part of $P^{*}$ going from $v_{1}$ to $v_{k}$ and the part of $P_{r+1}$ going from $v_{k}$ to $x$. The length of $P$ is greater than or equal to $q+r$ (there are $q$ edges from $v_{1}$ to $v_{q+1}$ and at least $r$ edges from $v_{q+1}$ to $x$, since $\left.d\left(x, v_{q+1}\right)=r\right)$. So $P$ is again longer than $P^{*}$, a contradiction.

So we have seen that in all cases, $P^{*}$ contains at least $2 r+1$ vertices.

As an immediate consequence, we obtain a result which was proved in [12].

Corollary 2 [12, Prop. 4.1] Let $r \geq 1$ and $G$ be any connected $r$-twin-free graph with $n>1$ vertices. Then we have $n \geq 2 r+1$.

The bound of Corollary 2 is the best possible, since, for any $r \geq 1$, the paths $P_{n}$ are $r$-twin-free for any $n \geq 2 r+1$ (see [2] for a study of $r$-identifying codes on paths). We conjecture that a statement stronger than that in Theorem 1 holds:

Conjecture 3 Let $r \geq 1$ and $G$ be any connected $r$-twin-free graph with at least two vertices. Then $P_{2 r+1}$ is an induced subgraph of $G$.

This conjecture is true for $r=1$, as we now show, using the following lemma.

Lemma 4 Let $r \geq 1$ and $G$ be any connected $r$-twin-free graph with at least two vertices. Then $P_{r+2}$ is an induced subgraph of $G$.

Proof. Consider two distinct vertices $a$ and $b$ with $d(a, b)=1$. Since they are not twins, there exists (without loss of generality) a vertex $x(x \neq b)$ such that $x \in B_{r}(b)$ and $x \notin B_{r}(a)$. Because $a$ and $b$ are adjacent, we have $d(b, x)=r$ and $d(a, x)=r+1$, which means that $a$ and any vertices forming a shortest path between $b$ and $x$ constitute a path with $r+2$ vertices and no chord.

Corollary 5 Let $G$ be any connected one-twin-free graph with at least two vertices. Then $P_{3}$ is an induced subgraph of $G$.

\section{Induced subgraphs with one vertex less}

Let $r \geq 1$ and $G=(V, E)$ be a connected, $r$-twin-free graph with at least two vertices; we say that $G$ is $r$-terminal if for all vertices $x \in V, G_{x}$ is not $r$-twin-free, and that $G$ is not $r$-terminal if there exists a vertex $x \in V$ such that $G_{x}$ is $r$-twin-free. We denote by $\mathcal{T}_{r}$ the set of $r$-terminal graphs.

Thanks to Corollary 2, we need only to consider graphs with $n \geq 2 r+1$. Using Theorem 1, it is easy to see that if $n=2 r+1$, the only $r$-twin-free graph is the path $P_{2 r+1}$, for $r \geq 1$, and the only $r$-terminal graph is $P_{2 r+1}$, for $r>1$ (the case of $P_{3}$ is particular, because removing the middle vertex yields two isolated vertices which constitute a onetwin-free graph — see Remark 2).

In this section, we address the following issue: are the paths $P_{2 r+1}$ (with the exception of $P_{3}$ ) the only $r$-terminal graphs? 
The answer to this question is multifold: it is positive if $r=1$ (Corollary 7), or if we restrict ourselves, for any $r$, to trees (Corollary 11); it is negative if $r \geq 3$ (Theorem 12). The case $r=2$ remains open.

\subsection{The case $r=1$}

The following lemma can be found in [1], [9], [8].

Lemma 6 Let $n \geq 3$ be an integer, and $G$ be any connected one-twin-free graph with $n$ vertices. Then there exists a one-identifying code in $G$ with $n-1$ vertices.

Proof. We refer to [9], which gives an elegant proof of a more general result.

An easy consequence of Lemma 6 is that $\mathcal{T}_{1}=\emptyset$ :

Corollary 7 Let $n \geq 3$ be an integer, and $G=(V, E)$ be any connected one-twin-free graph with $n$ vertices. Then $G$ is not one-terminal.

Proof. If $n=3, G=P_{3}$, so we can assume that $n \geq 4$. By Lemma 6 , there is a one-identifying code $C$ of size $n-1$ in $G$. Consider $G_{x}$ with $\{x\}=V \backslash C\left(G_{x}\right.$ may be connected or not); obviously, $C$ is still one-identifying in $G_{x}$, because removing $x$ does not cut connexions of length $r(=1)$ between pairs of vertices not containing $x$ itself (this explains why the cases $r=1$ and $r>1$ are different). Therefore, $G_{x}$ is one-twin-free.

The following theorem sharpens Corollary 7.

Theorem 8 Let $n \geq 4$ be an integer, and $G=(V, E)$ be any connected one-twin-free graph with $n$ vertices. Then there exists a vertex $x \in V$ such that $G_{x}$ is one-twin-free and connected.

Proof. In this proof, we use twin, twin-free and terminal for one-twin, one-twin-free and one-terminal, respectively. By Corollary 7 , we know that $G$ is not terminal. If Theorem 8 were not true, let $G=(V, E)$ be the smallest counter-example, that is, $G$ satisfies:

(i) $G$ is connected,

(ii) $G$ is twin-free,

(iii) for all $x \in V, G_{x}$ twin-free $\Rightarrow G_{x}$ not connected,

(iv) $n \geq 4$, and

(v) among all graphs satisfying (i)-(iv), $|V|$ is the smallest possible.

We show that such a graph cannot exist.

Let $x \in V$ be such that $G_{x}$ is twin-free (such a vertex $x$ exists because $G$ is not terminal). By (iii), $G_{x}$ consists of at least two connected components, $F$ and $H$, see Figure 3.

If $G$ is a star centered at $x$ with at least four vertices, i.e., $G=(V, E)$ where $V=$ $\left\{x, v_{1}, \ldots, v_{n-1}\right\}, E=\left\{\left\{x, v_{i}\right\}: 1 \leq i \leq n-1\right\}, 4 \leq n$, then for any $i$ between 1 and $n-1$, $G_{v_{i}}$ is twin-free and connected, contradicting (iii). Threrefore we assume from now on that at least one connected component in $G_{x}$, say $H$, has at least two vertices. 


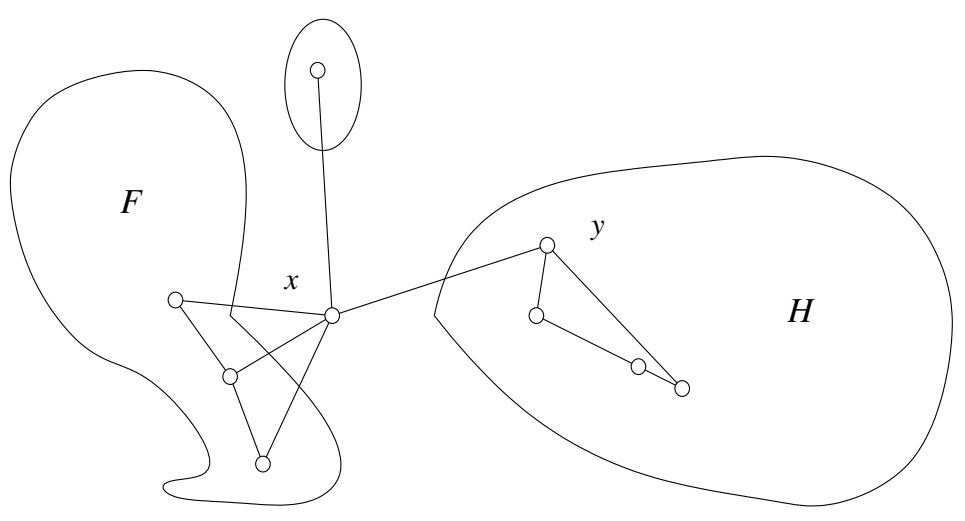

Figure 3: The vertex $x$ and the connected components of $G_{x}$.

Step 1. We show that there are at least two edges between $x$ and $H$. Assume on the contrary that there is only one, say $\{x, y\}$ (see Figure 3 ).

We construct the graph $G^{\prime}=\left(V^{\prime}, E^{\prime}\right)$ by contracting the vertices $x$ and $y$ into one vertex $x y: V^{\prime}=(V \backslash\{x, y\}) \cup\{x y\}, E^{\prime}=(E \backslash\{\{x, y\}\} \backslash\{\{x, t\}:\{x, t\} \in E\} \backslash\{\{y, t\}$ : $\{y, t\} \in E\}) \cup\{\{x y, t\}:\{x, t\} \in E$ or $\{y, t\} \in E\}$.

It is easy to see that $G^{\prime}$ is connected and twin-free, because $G$ is. We now show that $G^{\prime}$ satisfies (iii). Let $z \in V^{\prime}$ be such that $G_{z}^{\prime}$ is twin-free (such a $z$ exists, because, by Corollary $7, G^{\prime}$ is not terminal).

If $z=x y$, then $G_{z}^{\prime}$ is not connected.

If $z \neq x y, G_{z}$ is twin-free, because $G_{z}^{\prime}$ is. Then, by (iii), $G_{z}$ is not connected. This in turn implies that $G_{z}^{\prime}$ is not connected.

Therefore $G^{\prime}$ satisfies (i)-(iii) and has fewer vertices than $G$, a contradiction, unless $n=4$. In this case however, we would have $G^{\prime}=P_{3}$ and necessarily, since $G$ is twin-free, $G=P_{4}$, but $P_{4}$ does not satisfy (iii).

This proves that there are at least two edges between $x$ and $H$. This also shows that there are at least three vertices in $H$ : if $y$ and $z$ were the only vertices in $H$ and since they are connected, they would be twins (both in $G$ and $G_{x}$ ).

Step 2. We still consider the connected component $H$, which by assumption is twin-free and has at least three vertices.

If $H$ has exactly three vertices, then $H=P_{3}$ and it is easy to see from Figure 4 that, no matter how the vertices in $H$ are linked to $x$ in $G$, it is possible to find a vertex $u$ in $H$ such that $G_{u}$ is twin-free and connected, again contradicting (iii).

If $H$ has at least four vertices, then, since $H$ has fewer vertices than $G, H$ cannot satisfy simultaneously (i)-(iii). But $H$ is connected and twin-free, by assumption. So $H$ does not satisfy (iii): there is a vertex $u$ in $H$ such that $H_{u}$ is connected and twin-free. It is not difficult now to see that $G_{u}$ is connected and twin-free, again contradicting (iii). Indeed, $G_{u}$ is connected because $x$ is connected to a vertex other than $u$ in $H$, as seen in Step 1; and $G_{u}$ is twin-free, because $H_{u}$ and $G_{x}$ are twin-free and obviously $x$ is not a twin in $G_{u}$. 


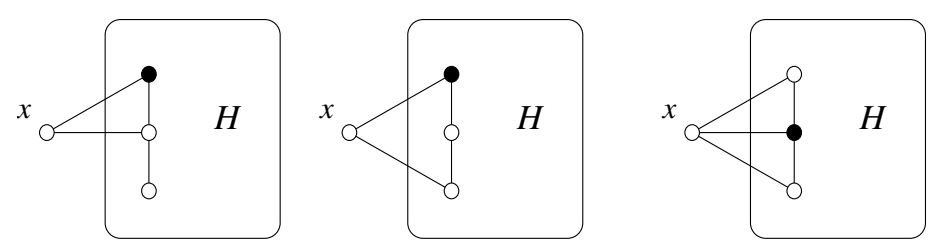

Figure 4: The case $H=P_{3}$. Vertices in black can be removed.

In all cases, we see that a graph $G$ satisfying (i)-(iv) does not exist, which proves Theorem 8.

\subsection{Trees}

We now consider the case of trees. We first give an easy lemma.

Lemma 9 Let $r \geq 1$ and $n \geq 2 r+2$ be integers, and $T=(V, E)$ be any (connected) $r$-twin-free tree with $n$ vertices. If $\beta \in V$ has degree at least three, and if $C_{1}, C_{2}, \ldots, C_{p}$ are the connected components of $T_{\beta}$, then at least $p-1$ components $C_{i}$ have, as an induced subgraph, a path with at least $r$ vertices which has one end adjacent to $\beta$.

Proof. Let $c_{i, 1} \in C_{i}$ be at distance one from $\beta$. Since the $p$ sets $B_{r}\left(c_{i, 1}\right)$ are different and nonempty, at least $p-1$ of the vertices $c_{i, 1}$ have a vertex at distance at least $r-1$ in $C_{i}$.

In other words, we have just proved that at least $p-1$ components contain (at least) one leaf $f$ (that is, a vertex with degree one) with $d(f, \beta) \geq r$.

Theorem 10 Let $r \geq 1$ and $n \geq 2 r+2$ be integers, and $T=(V, E)$ be any (connected) $r$-twin-free tree with $n$ vertices. Then there exists a leaf $x \in V$ such that $T_{x}$ is $r$-twin-free (and connected).

Proof. If $T$ is a path, removing one of its ends gives a path with at least $2 r+1$ vertices, which is still $r$-twin-free. So we assume that there is at least one vertex with degree at least three.

In the rest of this proof, we shall say that a vertex separates two vertices $v_{1}$ and $v_{2}$ if it covers exactly one of them, which means that $v_{1}$ and $v_{2}$ are not twins.

For any leaf $\alpha \in V$, let $\beta_{\alpha}$ be its closest vertex with degree at least three; among all leaves, we choose a leaf which has the smallest possible $d\left(\alpha, \beta_{\alpha}\right)$. We call this leaf $x$, we set $d=d\left(x, \beta_{x}\right)$ (so the distance between any leaf and any vertex with degree at least three is at least $d$ ), and we claim that $T_{x}$ is $r$-twin-free. Since all the vertices that are $r$-covered by $x$ are also $r$-covered by the (only) vertex at distance one from $x$, all we have to show is that, if $x r$-separates two vertices, $v_{1}$ and $v_{2}$, belonging to $V \backslash\{x\}$, then there is another vertex, $z$, that also separates them. For the same reason, without loss of generality, we can assume that $d\left(x, v_{1}\right) \leq r$ and $d\left(x, v_{2}\right)$ is equal to $r+1$ (otherwise, 
(a)

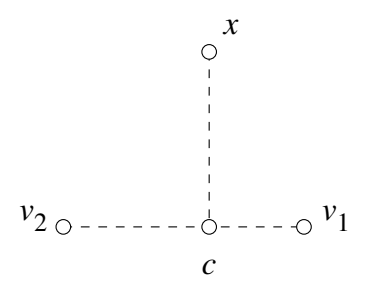

(b)

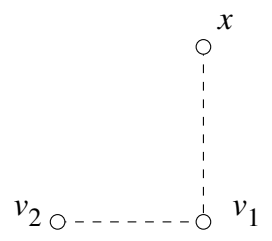

Figure 5: How to find a vertex $x$ such that $T_{x}$ is twin-free (1).

(a)(i)

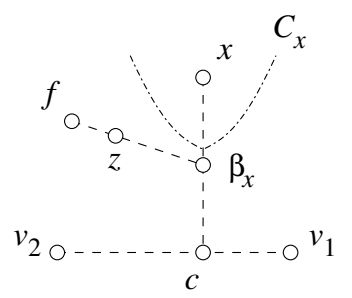

(a)(ii)

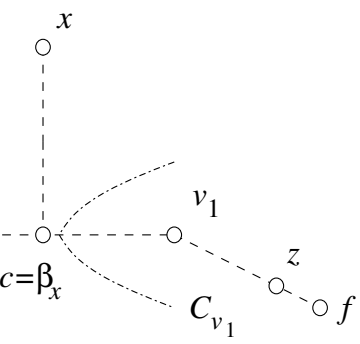

Figure 6: How to find a vertex $x$ such that $T_{x}$ is twin-free (2).

the vertex at distance one from $x$ would separate $v_{1}$ and $v_{2}$ ). We can also assume that $d\left(v_{1}, v_{2}\right) \leq r$ : otherwise, $v_{1} \notin B_{r}\left(v_{2}\right)$ and $v_{1}$ and $v_{2}$ are not twins.

Consider the two paths between $x$ and $v_{1}$ on the one hand, $x$ and $v_{2}$ on the other hand. Two cases occur, depending on whether they both contain $v_{1}$ or not (see Figure 5).

Case (a): the two paths diverge before reaching $v_{1}$. Let $c$ be the vertex where the two paths diverge; it is clear that $\beta_{x}$ is between $x$ and $c$. Two subcases occur: (i) $\beta_{x} \neq c$, (ii) $\beta_{x}=c$ (see Figure 6).

(i) $\beta_{x} \neq c$ : from now on, for a vertex $y \in V \backslash\left\{\beta_{x}\right\}$, let $C_{y}$ be the connected component containing $y$ in $T_{\beta_{x}}$. By the definition of $\beta_{x}$ and $d$, there is a connected component in $T_{\beta_{x}}$, different from $C_{x}$ and $C_{c}$, which contains a leaf $f$ with $d\left(f, \beta_{x}\right) \geq d$. This shows that on this path, the vertex $z$ at distance $d$ from $\beta_{x}$ plays the same role as $x$ with respect to $v_{1}$ and $v_{2}: z r$-covers $v_{1}$, not $v_{2}$.

(ii) $\beta_{x}=c$ : then in $C_{v_{1}}$, there is at least one leaf, $f$, which is linked to $\beta_{x}$ by a path going through $v_{1}$, and we know that $d\left(f, \beta_{x}\right) \geq d$. On this path, the vertex $z$ at distance $d$ from $\beta_{x}$ plays a role similar to $x$ : it $r$-covers $v_{1}$, not $v_{2}$.

Case (b): the path going to $v_{2}$ goes through $v_{1}$. There are four subcases: (i) $\beta_{x}$ is between $x$ and $v_{1}$, (ii) $\beta_{x}$ is between $v_{1}$ and $v_{2}$ with $d\left(\beta_{x}, v_{1}\right) \neq d\left(\beta_{x}, v_{2}\right)$, (iii) $\beta_{x}$ is between $v_{1}$ and $v_{2}$ with $d\left(\beta_{x}, v_{1}\right)=d\left(\beta_{x}, v_{2}\right)$, (iv) $\beta_{x}$ is on the other side of $v_{2}$ (see Figure 7).

(i) $\beta_{x}$ is between $x$ and $v_{1}$ (and $\beta_{x}$ can be equal to $v_{1}$ ):

this case is actually the same as Case (a)(i), with $v_{1}=c$ : let $f$ be a leaf in a connected component in $T_{\beta_{x}}$ which is neither $C_{x}$ nor $C_{v_{2}}$. Again, $d\left(f, \beta_{x}\right) \geq d$, and between $\beta_{x}$ and $f$, there is a vertex $z$ at distance $d$ from $\beta_{x}$, which plays the same role as $x$. 
(b)(i)
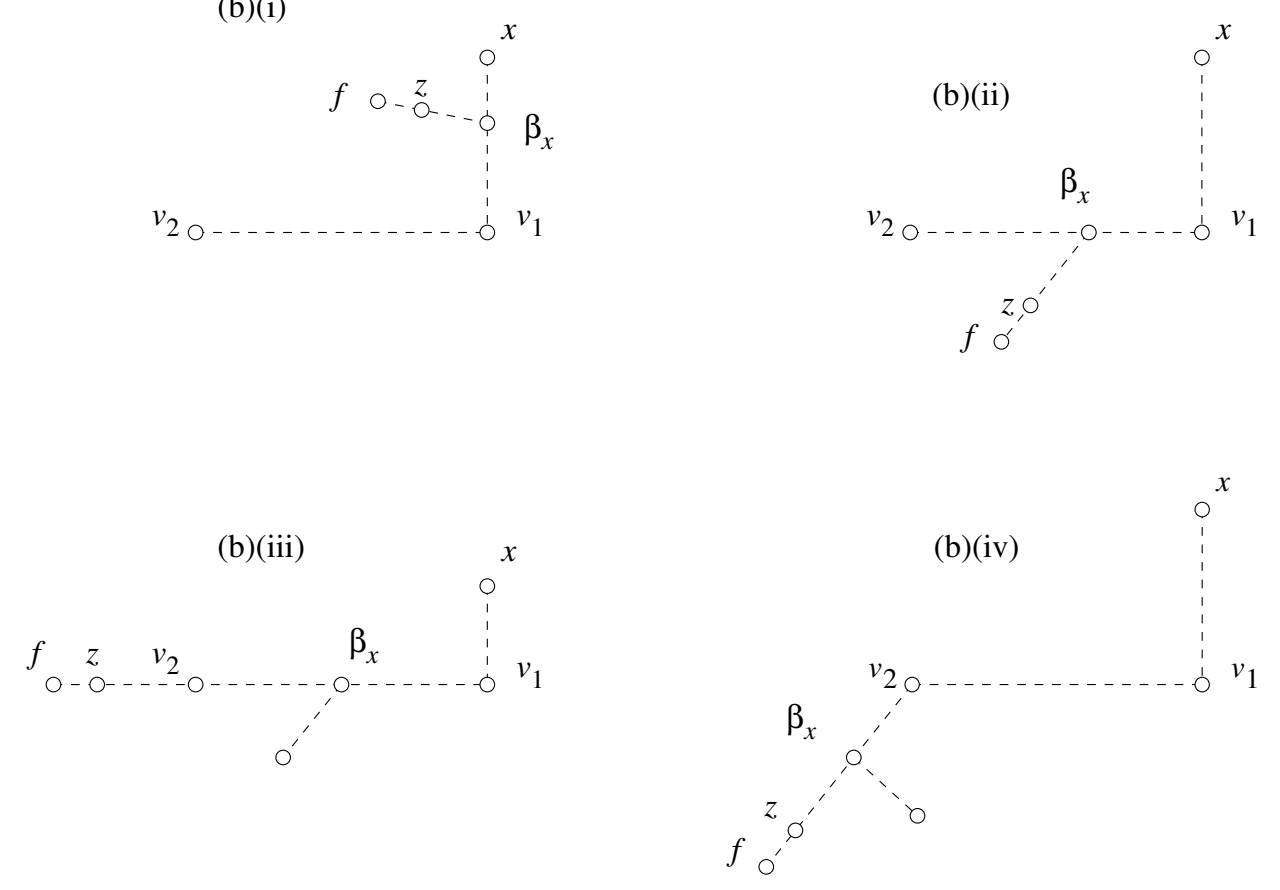

Figure 7: How to find a vertex $x$ such that $T_{x}$ is twin-free (3).

(ii) $\beta_{x}$ is between $v_{1}$ and $v_{2}$, with $d\left(\beta_{x}, v_{1}\right) \neq d\left(\beta_{x}, v_{2}\right)$ (and $\beta_{x}$ can be equal to $v_{2}$, not to $\left.v_{1}\right)$ :

among the connected components in $T_{\beta_{x}}$ which are not $C_{v_{1}}\left(\right.$ if $v_{2}=\beta_{x}$ ), and are neither $C_{v_{1}}$ nor $C_{v_{2}}$ (if $v_{2} \neq \beta_{x}$ ), let $f$ be a leaf with the property that the distance between $f$ and $\beta_{x}$ is the largest possible. If $d<r$, then by Lemma $9, d\left(f, \beta_{x}\right) \geq r$; if $d \geq r$, then $d\left(f, \beta_{x}\right) \geq r$, because $d\left(f, \beta_{x}\right) \geq d$. Then, since $d\left(v_{1}, v_{2}\right) \leq r$, it is easy to find, between $f$ and $\beta_{x}$, a vertex $z$ which $r$-covers $v_{1}$, not $v_{2}$ (respectively, $v_{2}$, not $\left.v_{1}\right)$, if $d\left(\beta_{x}, v_{1}\right)<d\left(\beta_{x}, v_{2}\right)$ (respectively, $\left.d\left(\beta_{x}, v_{1}\right)>d\left(\beta_{x}, v_{2}\right)\right)$, i.e., a vertex other than $x$ which also separates $v_{1}$ and $v_{2}$.

(iii) $\beta_{x}$ is between $v_{1}$ and $v_{2}$, with $d\left(\beta_{x}, v_{1}\right)=d\left(\beta_{x}, v_{2}\right)=\delta$ :

let $f$ be a leaf on the other side of $v_{2}$; again, $d\left(f, \beta_{x}\right) \geq d=\delta+d\left(x, v_{1}\right)>\delta$. Let $z$ be the vertex between $f$ and $v_{2}$ at distance $d$ from $\beta_{x}$. Then $d\left(x, v_{1}\right)=d\left(z, v_{2}\right)$ and $d\left(x, v_{2}\right)=d\left(z, v_{1}\right)$, which means that $z$ also separates $v_{1}$ and $v_{2}$.

(iv) $\beta_{x}$ is on the other side of $v_{2}$ (and is not equal to $v_{2}$ ):

by Lemma 9 , there is a leaf $f$ in a connected component in $T_{\beta_{x}}$ which is not equal to $T_{v_{2}}$, which is at distance at least $r$ from $\beta_{x}$. Between $f$ and $v_{2}$, there is a vertex $z$ at distance $r$ from $v_{2}$, which therefore separates $v_{2}$ and $v_{1}$.

In all cases, there is a vertex $z$, other than $x$, which separates $v_{1}$ and $v_{2}$. Therefore, $G_{x}$ is twin-free. Moreover, since $x$ is a leaf, $G_{x}$ is connected.

We therefore have the following corollary, the first part of which is already contained in Corollary 7. 
Corollary 11 There is no one-terminal tree. For a given $r>1$, the only $r$-terminal tree is the path $P_{2 r+1}$.

\subsection{The case $r \geq 3$}

We now consider general graphs, for $r \geq 3$.

Theorem 12 For each integer $r \geq 3$, there is a graph $G, G \neq P_{2 r+1}$, which is $r$-terminal.

Proof. We search for a connected $r$-twin-free graph $G=(V, E)$, with $|V| \geq 2 r+2, r \geq 3$, such that for all $x \in V, G_{x}$ is not $r$-twin-free.

In this proof, calculations are carried modulo $2 r$. Take a cycle of length $2 r$ with vertices $c_{i}(i=0,1, \ldots, 2 r-1)$, and add one vertex $s_{i}$ (which we shall call the spike of $c_{i}$ ) together with the edge $\left\{c_{i}, s_{i}\right\}$, for every value of $i$ except one.

The resulting graph $G$ is clearly $r$-twin-free. A cycle point $c_{i} r$-covers all the vertices except the spike $s_{i+r}$ diagonally across - and of course there is one cycle point which $r$-covers all the vertices in the graph. In particular, each point in the cycle $r$-covers all the vertices in the cycle. Each spike $s_{i} r$-covers all the cycle points except $c_{i+r}$ (the one diagonally across); so, indeed, the graph is $r$-twin-free.

It is also $r$-terminal. If we remove one spike, then there is another cycle point that $r$-covers all the vertices in the graph. If we consider one cycle point, $c_{i}$, then (since $r \geq 3$ ), both $c_{i+1}$ and $c_{i+2}$ - or both $c_{i-1}$ and $c_{i-2}$ - have spikes; say, $s_{i+1}, s_{i+2}$ are in the graph $G$. If now we remove $c_{i}$, then $c_{i+1}$ and $s_{i+2}$ trivially $r$-cover the same vertices because $r \geq 3$.

Other constructions can be thought of. We give, without proof, one example which gives smaller graphs than the ones in the proof of Theorem 12. In this example, calculations are carried modulo $2 r+1$. We consider a cycle of length $2 r+1(r \geq 3)$ with vertices $c_{i}$ $(i=0,1, \ldots, 2 r)$, and add the spike $s_{i}$ for every value of $i$ except

- $3 k$ and $2 r+1-3 k$, for $0 \leq k \leq m$, if $r=3 m$ or $3 m+1$,

- $2+3 k$ and $2 r+1-(2+3 k)$, for $0 \leq k \leq m$, if $r=3 m+2$,

see Figure 8 for $r \in\{3,4,5,6\}$.

\subsection{Large terminal graphs and open problems}

The above example as well as the construction of Theorem 12 do not work for $r=2$. Other constructions have been tested and failed, and the problem remains open: apart from $P_{5}$, do two-terminal graphs exist?

When $r \geq 6$, the set $\mathcal{T}_{r}$ is infinite, as shows the following theorem.

Theorem 13 For each integer $r \geq 6$, there are infinitely many $r$-terminal graphs.

Proof. Assume first that $r \geq 7$.

For each $i=1,2, \ldots, m(m \geq 3)$ let $\mathcal{C}_{i}$ be a $2 r$-cycle with vertices $c_{i}(j)$ (indices $j$ modulo $2 r$ ). Connect the cycles together by adding an edge from $c_{i}(r)$ to $c_{i+1}(0)$ for all 

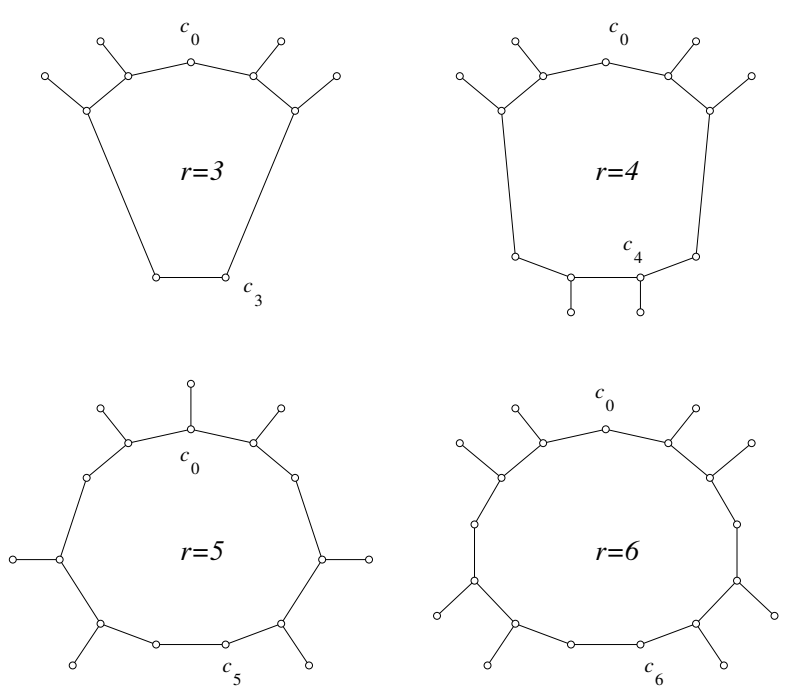

Figure 8: Different $r$-terminal graphs.

$i=1,2, \ldots, m-1$ and from $c_{m}(r)$ to $c_{1}(0)$; in fact, it is convenient to consider indices $i$ modulo $m$.

For all $i$ add spikes $s_{i}(j)$ to all the vertices $c_{i}(j)$ with $j=1,-2,3,-4, \ldots,(-1)^{r}(r-1)$. There are $m(r-1)$ such vertices, see Figure 9 .

We claim that this graph $G$ with $n=m(3 r-1)$ vertices is $r$-terminal.

We first prove that $G$ is $r$-twin-free. Assume that we know $B_{r}(v)$ for an unknown vertex $v$. We show that we can deduce $v$.

Clearly, $\mathcal{C}_{i} \subseteq B_{r}(v)$ if and only if $v \in \mathcal{C}_{i}$. Assume that this is the case. For $j=$ $0,1, \ldots, r-1$, the vertices $c_{i}( \pm j)$ both $r$-cover $2 r-1-2 j$ vertices of $\mathcal{C}_{i-1}$, and $c_{i}(r)$ does not cover any vertices of $\mathcal{C}_{i-1}$, and, moreover, for $j= \pm 1, \pm 2, \ldots, \pm(r-1)$, exactly one of $c_{i}(j+r)$ or $c_{i}(-j-r)$ (the points diagonally opposite to $c_{i}(j)$ and $\left.c_{i}(-j)\right)$ has a spike attached to it and this spike $r$-separates $c_{i}(j)$ and $c_{i}(-j)$, so we can identify $v$.

If $v$ does not belong to any $\mathcal{C}_{i}$, then it is one of the spikes. Given $B_{r}(v)$, we find out which is the cycle $\mathcal{C}_{i}$ of which $B_{r}(v)$ contains $2 r-1$ vertices (there is only one). If the vertex of $\mathcal{C}_{i}$ which is missing from $B_{r}(v)$ is $c_{i}(j)$, then we know that $v$ is the spike diagonally opposite, i.e., $v=s_{i}(j+r)$.

It suffices now to prove that $G$ is $r$-terminal. If we delete one of the spikes, say $s_{i}(j)$, then by the construction $s_{i}(-j)$ is not a spike either, and $c_{i}(j+r)$ and $c_{i}(-j-r)$ are $r$-twins.

Assume finally that we delete a vertex $c_{i}(j) \in \mathcal{C}_{i}$ for some $i$ and $j$.

If $j=0$, then $c_{i}(-1)$ and $s_{i}(-2)$ are twins. The case $j=r$ is symmetrical.

Assume that $j \in S:=\{ \pm 1, \ldots, \pm(r-1)\}$. Because $r \geq 7$, we know that $j-1, j-$ $2, j-3 \in S$ or $j+1, j+2, j+3 \in S$; say the latter. By the construction, $c_{i}(j+2)$ has a spike or $c_{i}(j+1)$ and $c_{i}(j+3)$ both have spikes. If $c_{i}(j+2)$ has a spike, then $c_{i}(j+1)$ and $s_{i}(j+2)$ are twins; if $c_{i}(j+1)$ and $c_{i}(j+3)$ both have spikes, then $c_{i}(j+2)$ and $s_{i}(j+3)$ are twins. 

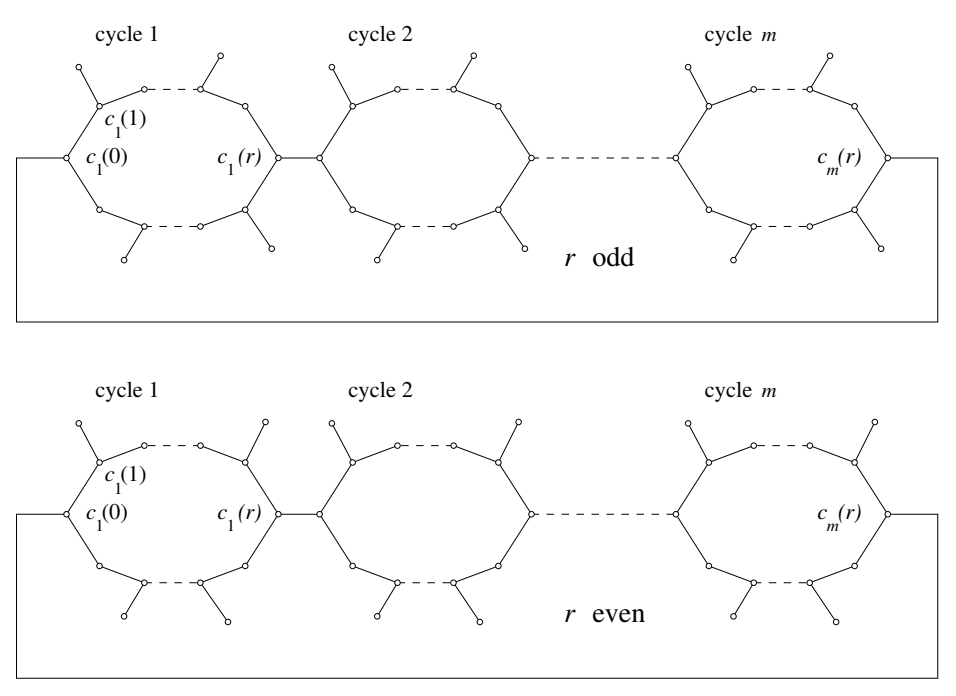

$r>6$

Figure 9: The $r$-terminal graph constructed in the proof of Theorem 13.

Assume finally that $r=6$. We can use the same argument, if we add spikes to $c_{i}(j)$ with $j=1,-2,-3,4,-5$ (instead of what we did in the general case).

Therefore, another open problem is the situation for $r=3,4,5$ : there exist $r$-terminal graphs, but are they in finite or infinite number?

Observe that, whether they are in finite or infinite number, if we could prove that, for a given $r>1$, all $r$-terminal graphs contain the path $P_{2 r+1}$ as an induced subgraph (which is the case for all $r$-terminal graphs described in Section 3.3), then Conjecture 3 would hold: simply consider a graph $G$ which is not $r$-terminal, and delete vertices in $G$ until you get a graph $G^{\prime}$ which is $r$-terminal; if $G^{\prime}$ contains $P_{2 r+1}$ as an induced subgraph, so does the initial graph $G$.

\section{References}

[1] N. Bertrand, Codes identifiants et codes localisateurs-dominateurs sur certains graphes, Mémoire de stage de maîtrise (under O. Hudry's supervision), ENST, Paris (France), June 2001.

[2] N. Bertrand, I. Charon, O. Hudry, A. Lobstein, Identifying and locating-dominating codes on chains and cycles, Europ. J. Combin., 25/7 (2004), 969-987.

[3] N. Bertrand, I. Charon, O. Hudry, A. Lobstein, 1-identifying codes on trees, Austral. J. Combin., 31 (2005), 21-35.

[4] U. Blass, I. Honkala, S. Litsyn, Bounds on identifying codes, Discrete Math. 241 (2001), 119-128.

[5] I. Charon, O. Hudry, A. Lobstein, Identifying codes with small radius in some infinite regular graphs, Electron. J. Combin. 9(1) (2002), R11. 
[6] I. Charon, O. Hudry, A. Lobstein, Minimizing the size of an identifying or locatingdominating code in a graph is NP-hard, Theoret. Comput. Sci. 290(3) (2003), 21092120 .

[7] I. Charon, O. Hudry, A. Lobstein, On the structure of identifiable graphs, Electron. Notes Discrete Math. 22 (2005), 491-495.

[8] I. Charon, O. Hudry, A. Lobstein, Extremal cardinalities for identifying and locatingdominating codes in graphs, Discrete Math., 307 (2007), 356-366.

[9] S. Gravier, J. Moncel, On graphs having a $V \backslash\{x\}$ set as an identifying code, Discrete Math., 307 (2007), 432-434.

[10] I. Honkala, T. Laihonen, S. Ranto, On strongly identifying codes, Discrete Math. 254 (2002), 191-205.

[11] M. G. Karpovsky, K. Chakrabarty, L. B. Levitin, On a new class of codes for identifying vertices in graphs, IEEE Trans. Inform. Theory 44 (1998), 599-611.

[12] J. Moncel, Codes identifiants dans les graphes, Thèse de Doctorat de l'Université de Grenoble, France, 2005.

[13] S. Ranto, I. Honkala, T. Laihonen, Two families of optimal identifying codes in binary Hamming spaces, IEEE Trans. Inform. Theory 48 (2002), 1200-1203.

[14] http://www.infres.enst.fr/ lobstein/bibLOCDOMetID.html 